
\title{
25 Research Square \\ Effects of Sleep Disturbance on Neuropsychological Functioning in Patients with Pediatric Brain Tumor
}

\author{
Jenna A. Chiang
}

Children's Hospital of Los Angeles

\section{Paulina T. Feghali}

Children's Hospital of Los Angeles

Anita Saavedra

Children's Hospital of Los Angeles

Ashley M. Whitaker ( $\square$ ashleymarie.whitaker@gmail.com )

Children's Hospital Los Angeles https://orcid.org/0000-0002-4362-6295

\section{Research Article}

Keywords: Sleep, Brain tumor, Adaptive functioning, Executive functioning, Patient reported outcome (PRO)

Posted Date: December 1st, 2021

DOI: https://doi.org/10.21203/rs.3.rs-1126325/v1

License: (c) (1) This work is licensed under a Creative Commons Attribution 4.0 International License. Read Full License

Version of Record: A version of this preprint was published at Journal of Neuro-Oncology on January 29th, 2022. See the published version at https://doi.org/10.1007/s11060-022-03954-4. 


\section{Abstract}

Purpose While the effects of sleep on cognition in typically developing children are well established, there is a paucity of research in patients with pediatric brain tumor (PBT), despite their increased risk for sleeprelated disturbances. The aim of this study was to examine the impact of sleep factors on patientreported outcome (PRO) measures, including adaptive and executive functioning within this population.

Methods 133 patients with PBT (52\% male) ages 5-23 ( $\overline{\mathrm{x}}=12.8 \mathrm{yrs} ; S D=4.5 \mathrm{yrs})$ underwent neuropsychological evaluation, including assessment of adaptive and executive functioning. Subjective sleep concerns, nocturnal sleep duration, and daytime sleep behavior were also collected and compared to age-based guidelines.

Results Nearly $30 \%$ of patients reported subjective sleep concerns, while the sample as a whole presented with reduced nocturnal sleep duration (approximately one hour below age-based recommendations). Despite the expectation for monophasic sleep by age five, nearly half of the sample reported consistent daytime napping. With regard to functional outcomes, inadequate sleep predicted decreased adaptive functioning, $\mathrm{F}(1,56)=4.23, p<.05\left(R^{2}=.07\right)$, as well as increased symptoms of executive dysfunction, $\mathrm{F}(1,108)=3.51, p<.05\left(R^{2}=.03\right)$.

Conclusion Patients with PBT demonstrate several aspects of abnormal sleep, which are associated with poorer long-term PROs. Further exploration of diagnostic, treatment-related, and demographic variables will be needed to better understand these relationships among patients with PBT in order to inform appropriate interventions.

\section{Introduction}

Sleep is an essential component to daily functioning and is necessary for multiple aspects of development, as well as energy conservation [1] and brain waste clearance [2]. Sleep also helps modulate cognition, disease, immune response, and psychological state [1-3]. Although adequate sleep is known to be imperative for optimal functioning, average sleep duration (i.e., length of sleep) from infancy through adolescence has steadily declined within the general population over the last 20 years $[4,5]$. The consequences of insufficient sleep or reduced sleep duration in children have been associated with a wide range of adverse physical and mental health outcomes [5], as well as deficits in cognition [6-10], and executive functioning (EF; higher order thinking skills) in particular [9]. Increased risk and incidence of psychiatric disorders are also observed [11,12], as sleep and mental health appear to be bidirectional processes [13]. Accordingly, sleep difficulties in children are associated with poorer quality of life and secondary familial effects (e.g., maternal stress) [14]. Sex and race/ethnicity are important factors to examine as well, considering prior studies reveal that girls consistently demonstrate greater sleep duration and shorter sleep latency (i.e., amount of time to fall asleep) than boys [15], while ethnic/racial minority [16] is identified as a risk factor for disrupted sleep. 
While daytime napping is important for early cognition, transition to monophasic sleep by age five is a developmental marker of brain maturation [17]. As children age, lower sleep quality, longer sleep latency, more arousals, and increased time spent in lighter sleep may be associated with napping behaviors due to circadian misalignment, as the light-dark cycle and endogenous circadian rhythms are at risk of deviation [18]. Additionally, according to the two-process model of sleep regulation, napping behaviors can interfere with circadian regulatory processes by delaying accumulation of sleep pressure necessary for successful sleep initiation [19].

Chronic and acute medical conditions increase the risk of sleep disruption in children [20]. Pediatric cancer and associated treatments significantly interfere with multiple aspects of sleep, such as sleep latency [21], sleep duration [22], daytime sleepiness [23], sleep habits (e.g., disturbed sleep-wake rhythm) [24], and fatigue [25]. Pediatric patients both with and without central nervous system involvement of their cancer experience greater disturbances related to sleep initiation and maintenance compared to typically developing peers [26]. However, patients with pediatric brain tumor (PBT) in particular are at increased risk for sleep-related deficits, as the location of the tumor (e.g., with disruption to the hypothalamic-pituitary region) and associated focal treatments (e.g., resection, cranial irradiation) may disrupt circadian rhythms [27-29]. Moreover, increased daytime sleepiness and longer sleep duration in survivors of PBT can persist years after treatment into adulthood [25].

Neurocognitive impairment (including executive dysfunction) has been found to be correlated with fatigue and sleep problems in long-term female survivors of childhood leukemia, though not with their male counterparts [30]. Additionally, recent investigation of survivors of PBT with neurocognitive complaints in Amsterdam revealed $48 \%$ presented with sleep problems, which was associated with parent-reported executive dysfunction as measured by the Behavior Rating Inventory of Executive Function (BRIEF) [31]. While the need for adequate sleep to function properly is well documented, the association between inadequate sleep and adaptive functioning deficits among patients with PBT has yet to be explored, despite poor adaptive functioning being a known late effect of pediatric cancer [32].

Given the nature of how sleep needs and patterns shift drastically throughout the developmental period, the primary goal of this retrospective exploratory study was to further investigate sleep factors (i.e., subjective sleep concerns, nocturnal sleep duration, daytime napping patterns) across different age ranges of patients with PBT, as well as examine the impact of these sleep factors on reported functioning.

\section{Methods}

\section{Patients}

Archival data were used to conduct a retrospective study investigating sleep and neuropsychological outcomes in patients with PBT ages five and older who underwent evaluation through the Cancer and Blood Disease Institute (CBDI) at Children's Hospital Los Angeles (CHLA) between September 2014 and 
February 2020. Exclusionary criteria included intellectual disability, stroke, extreme prematurity, autism spectrum disorder, and insufficient sleep data. The study was approved by the CHLA Institutional Review Board as part of a larger project on neuropsychological sequelae of pediatric cancer.

\section{Measures}

Sleep data (e.g., estimated sleep duration and presence of napping/sleep concerns) were collected through clinical interview and background history form. Age-based recommendations for sleep duration were obtained from the National Sleep Foundation (Table 1).

Patient-reported outcome (PRO) measures were selected for this study given their ecological validity, as they have been shown to be especially sensitive to functional impact of cancer-related impairment and capture the nuances of how neuropsychological concerns manifest in real-word environments [33]. The General Adaptive Composite (GAC) of the Adaptive Behavioral Assessment System - Third Edition (ABAS-3) and Global Executive Composite (GEC) of the BRIEF - Preschool Version, Second Edition, or Adult Version (BRIEF-P, BRIEF-2, BRIEF-A) were used to assess AF and EF, respectively. As is common practice, caregiver-report was used as a proxy for PRO depending on age. The $A B A S-3$ has a mean $(\overline{\mathrm{x}})$ Standard Score (SS) of 100, with a standard deviation (SD) of 15 , while BRIEF scores are presented as TScores $(\overline{\mathrm{x}}=50 ; S D=10)$ with higher scores reflecting increased concerns.

Table 1

The National Sleep Foundation's sleep duration recommendations based on age [34]

\begin{tabular}{|lll|}
\hline Age Group & Age Range & Sleep Duration (hours) \\
\hline Newborn & $0-3$ months & $14-17^{*}$ \\
\hline Infant & $4-11$ months & $12-15^{*}$ \\
\hline Toddler & $1-2$ years & $11-14^{*}$ \\
\hline Preschooler & $3-5$ years & $10-13^{*}$ \\
\hline School-Aged & $6-13$ years & $9-11$ \\
\hline Teenager & $14-17$ years & $8-10$ \\
\hline Young Adult & $18-25$ years & $7-9$ \\
\hline & * Includes nocturnal and daytime sleep for age ranges in which daytime naps are appropriate \\
\hline
\end{tabular}

\section{Statistical Analyses}

Analyses were conducted using IBM SPSS Statistics, Version 27. An alpha level of 0.05 was used to indicate statistical significance (with < .01 noted when applicable). Demographic, sleep-related, and PRO variables were analyzed using $\overline{\mathrm{x}}$ and $S D$ for continuous variables, as well as frequencies and percentages for categorical variables. Chi-square analyses were conducted to identify differences in categorical sleep factors based on demographic differences (e.g., sex, ethnicity, age group). One-sample t-tests were used 
to examine whether PRO measures differed from age expectations, while linear regression analyses were conducted to explore reduced nocturnal sleep duration as a predictor of significant functional concerns. Potential outliers were calculated using a 2.2 multiplier (one case was identified and excluded from associated analyses). Nonparametric analyses (Mann-Whitney, Kruskal Wallis) were utilized in the case of violated normality assumptions.

\section{Results}

\section{Demographic Variables}

A total of 133 patients ages 5 to 23 ( $\overline{\mathrm{x}}$ age $=12.8$ years; $S D=4.5$ years) were included in the dataset. Nearly $52 \%(n=69)$ identified as male and $\approx 48 \%(n=64)$ as female. Consistent with larger patient populations served at CHLA, the majority of the sample $(\approx 52 \%)$ identified as Latino $(n=69)$, followed by Caucasian ( $n=38 ; \approx 29 \%)$, Asian American $(n=11 ; 8 \approx \%)$, Black $(n=8 ; 6 \%)$, and Other $(n=7 ; \approx 5 \%)$. Tumor types and locations were variable (Table 2 ). There were no significant differences in reported sleep concerns, napping behaviors, or nocturnal sleep duration between sexes or ethnicities. 
Table 2

Sample Characteristics for Categorical Variables

\begin{tabular}{|lll|}
\hline Variable & Frequency & Percent \\
\hline Sex & & \\
\hline Male & 69 & 51.9 \\
\hline Female & 64 & 48.1 \\
\hline Race/Ethnicity & & \\
\hline White & 38 & 28.6 \\
\hline Latino & 69 & 51.9 \\
\hline Black & 8 & 6.0 \\
\hline Asian American & 11 & 8.3 \\
\hline Other & 7 & 5.3 \\
\hline Tumor Type & & \\
\hline Medulloblastoma & 30 & 22.6 \\
\hline Pilocytic Astrocytoma & 17 & 12.8 \\
\hline Germ Cell Tumor (Mixed) & 14 & 10.5 \\
\hline Optic Pathway Glioma & 14 & 10.5 \\
\hline Craniopharyngioma & 9 & 6.8 \\
\hline Anaplastic Ependymoma & 7 & 5.3 \\
\hline Astrocytic Tumor & 7 & 5.3 \\
\hline Atypical Teratoid/Rhabdoid Tumor & 5 & 3.8 \\
\hline Glioblastoma & 3 & \\
\hline Primitive Neuroectodermal Tumor & 3 & \\
\hline Other* & 24 & \\
\hline * Tumor Types with $n \leq 2$ & & \\
\hline
\end{tabular}

\section{PRO Measures}

As expected, overall AF was poorer than expected for patients with $\mathrm{PBT}$, with age-based normative scores just about one $S D$ below the mean on average ( $\overline{\mathrm{x}} \mathrm{GAC} S S=85.8$ ). A relatively similar downward trend in functioning was identified across $\mathrm{AF}$ domains, with the most noticeable concerns in practical $\mathrm{AF}(\overline{\mathrm{x}}$ 
Conceptual SS = 87.6; $\overline{\mathrm{x}}$ Social SS $=91.3, \overline{\mathrm{x}}$ Practical SS $=85.3)$. However, for the sample as a whole, no significant EF concerns were evident ( $\overline{\mathrm{x}} \mathrm{GEC}$ T-score $=51.5)$. See Table 3 for findings by age group.

Table 3

Sleep Concerns, Adaptive, and Executive Functioning By Age Group

\begin{tabular}{|c|c|c|c|c|c|c|c|}
\hline \multirow[t]{2}{*}{ Group } & \multirow[t]{2}{*}{$n$} & \multirow[t]{2}{*}{ Subjective Sleep Concerns } & \multirow[t]{2}{*}{ Napping } & \multicolumn{2}{|l|}{ GAC } & \multicolumn{2}{|l|}{ GEC } \\
\hline & & & & Mean & SD & Mean & SD \\
\hline $\begin{array}{l}\text { Preschoolers } \\
\text { Age } 5\end{array}$ & 7 & $17 \%$ & $57 \%$ & 95.3 & 3.0 & 48.4 & 8.1 \\
\hline $\begin{array}{l}\text { School Age } \\
\text { Ages 6-13 }\end{array}$ & 73 & $28 \%$ & $41 \%$ & 82.7 & 19.4 & 51.5 & 9.7 \\
\hline $\begin{array}{l}\text { Adolescents } \\
\text { Ages 14-17 }\end{array}$ & 33 & $21 \%$ & $55 \%$ & 90.3 & 17.9 & 50.6 & 8.6 \\
\hline $\begin{array}{l}\text { Young Adults } \\
\text { Ages 18-23 }\end{array}$ & 20 & $44 \%$ & $60 \%$ & 84.5 & 17.0 & 54.6 & 8.8 \\
\hline Percentages refer tc & the pe & centage of the sample reporting sleep & oncerns and d & ytime nap & & & \\
\hline GAC General Adapt & ve Con & osite, GEC Global Executive Composit & & & & & \\
\hline
\end{tabular}

\section{Subjective Sleep Concerns}

118 patients provided information regarding subjective sleep concerns and of those, nearly $28 \%$ reported concerns. Subjective sleep concerns were most notable among young adults (44\% of patients $18-23$ years of age) and least notable among preschoolers ( $17 \%$ of patients five years of age). However, no significant differences in reported sleep concerns were identified between age groups. See Table 3 for additional frequencies.

Patients with subjective sleep concerns were also reported to have increased executive dysfunction as compared to those without subjective sleep concerns $(U=573, p<.01)$. More specifically, EF for patients without subjective sleep concerns was noted to be average ( $\overline{\mathrm{x}}$ GEC T-score $=49.9)$, whereas those with subjective sleep concerns were approximately $2 / 3 S D$ above the mean ( $\bar{x}$ GEC T-score $=56.2)$. While this latter value does not exceed the BRIEF cutoff for clinical significance, it statistically exceeds normal levels in the general population $(t(109)=1.68, p<.05)$, and likely represents a trend toward increased executive 
dysfunction in these patients. While AF was lower than expected in the sample as a whole, there were no significant differences between patients with and without subjective sleep concerns.

\section{Nocturnal Sleep}

For the sample as a whole, reported nocturnal sleep duration was approximately 1 hour below National Sleep Foundation age recommendations ( $\overline{\mathrm{x}}=0.98$ hours). This varied significantly by age, $\mathrm{H}(3)=11.15, p$ $<.05$, with young adults obtaining the largest deviation from recommended sleep duration $(\overline{\mathrm{x}}=1.83$ hours less); however, this does not take into consideration daytime nap duration since typical young adults are expected to achieve monophasic sleep. Adolescents and school-aged children achieved 0.98 and 0.71 hours less than recommended for their ages, respectively. Nocturnal sleep was not interpretable for preschoolers based on age recommendations, which allow for daytime napping.

Nocturnal sleep and executive dysfunction were inversely related in that longer sleep duration predicted better $\mathrm{EF}$ within the sample as a whole, $\mathrm{F}(1,108)=3.51, p<.05\left(R^{2}=.03\right)$. Reduced duration also significantly predicted poorer general $\mathrm{AF}$ in the overall sample, $\mathrm{F}(1,56)=4.23, p<.05\left(R^{2}=.07\right)$. However, when further post-hoc analyses were conducted to compare patients with and without endorsed sleep concerns, these findings were only remarkable in conjunction with reported sleep disturbances for both $\mathrm{EF}, \mathrm{F}(1,23)=3.37, p<.05\left(R^{2}=.13\right)$, and $\mathrm{AF}, \mathrm{F}(1,12)=7.31, p<.01\left(R^{2}=.38\right)$. See Figures 1 and 2 .

\section{Daytime Napping}

65 patients (47\%) reported daytime napping, which is not considered developmentally appropriate after age five. There were no significant differences in napping behavior between age groups, though young adults (ages 18-23) notably had the largest frequency of daytime napping. See Table 3 for additional daytime napping frequencies.

\section{Discussion}

The purpose of this study was to investigate the effects of sleep on neuropsychological functioning in patients with PBT. Sleep factors (i.e., subjective sleep concerns, daytime napping, nocturnal sleep duration) across different age ranges within this population were explored, as well as their effect on reported $\mathrm{AF}$ and $\mathrm{EF}$ outcomes.

With regard to sleep factors, insufficient sleep duration was prevalent among patients with PBT. Reported subjective sleep concerns were found in nearly $28 \%$ of the sample, as compared to $20-25 \%$ in the general population [35]. Moreover, nearly half (47\%) of patients with PBT ages five and older were napping regularly, despite napping being developmentally atypical by this age [17]. Napping behavior did not vary significantly between age groups, as napping was more prevalent than expected across all age ranges; however, young adults (ages 18-23) surprisingly had the largest frequency of daytime napping (60\%). This is particularly interesting, as napping behaviors would be expected to decrease as one ages. 
Consistent with prior literature, there was a downward trend in AF for patients with PBT, particularly for the practical domain [32], as well as increased symptoms associated with executive dysfunction as compared to typically developing peers [31]. In terms of clinical significance, mean AF scores for this sample were nearly one $S D$ below the normative mean and did not differ between patients with and without reported sleep concerns, whereas EF scores were not clinically elevated (though approximately $2 / 3 S D$ above the mean for patients with subjective sleep concerns in particular). Inadequate sleep duration was associated with poorer $\mathrm{AF}$ and $\mathrm{EF}$ outcomes among patients with reported sleep concerns.

The above findings highlight the importance of addressing sleep-related concerns following diagnosis of PBT. Given increased risk for subjective sleep disturbances, as well as insufficient nocturnal sleep duration and increased need for daytime napping among this population, early identification of sleep problems can allow more timely provision of supports, such as appropriate referrals and therapies. Additionally, given the relationship between sleep factors and functional outcomes (both in terms of higher order thinking abilities and practical daily living skills), screening for sleep-related concerns can help inform treatment planning and ensure accommodations, modifications, and interventions to mitigate these sequelae.

As for limitations of the current study, the retrospective design did not allow predefined sleep data criteria, which restricted analyses and led to a more exploratory approach. Given the nuances of both organic and inorganic sleep disturbances, utilizing validated measures of sleep (e.g., Child Sleep Habits Questionnaire) in combination with direct measurement (e.g., actigraphy) is recommended to obtain sleep duration, latency, and maintenance. It would also be beneficial for future research to examine potential differences in tumor presentation (e.g., location), treatment-related factors (e.g., radiation), and additional demographic variables (e.g., socioeconomic status). More specifically, further investigation of factors contributing to the relationship between inadequate sleep and poor functional outcomes would help clarify future directions for identification and treatment.

\section{Conclusion}

Compared to the normative population, the above findings show patients with PBT present with shorter nocturnal sleep duration and greater occurrence of sleep concerns, with sleep factors associated with poorer adaptive and executive functioning (as reported on PRO measures). Future studies should aim to explore additional diagnostic, treatment, and demographic factors to better understand the association between sleep and neuropsychological outcomes in this population.

\section{Declarations}

Author contributions JAC and AMW designed the study. JAC and AS completed data collection. AMW performed statistical analyses. JAC, PTF, and AMW interpreted findings and prepared the manuscript. All authors approved final manuscript. 
Funding This research did not receive any specific grant from funding agencies in the public, commercial, or not-for-profit sectors.

Data availability Summary data supporting results reported in this article can be requested by emailing the senior author at awhitaker@chla.usc.edu.

Conflicts of interest The authors declare that they have no conflict of interest.

Ethical approval This retrospective study was approved by the institutional review board of Children's Hospital Los Angeles, Los Angeles, CA.

\section{References}

1. Zielinski, M. R., McKenna, J. T., \& McCarley, R. W. (2016). Functions and mechanisms of sleep. AIMS neuroscience, 3(1), 67-104. https://doi.org/10.3934/Neuroscience.2016.1.67

2. Xie, L., Kang, H., Xu, Q., Chen, M. J., Liao, Y., Thiyagarajan, M., O’Donnell, J., Christensen, D. J., Nicholson, C., Iliff, J. J., Takano, T., Deane, R., \& Nedergaard, M. (2013). Sleep drives metabolite clearance from the adult brain. Science, 6156, 373. https://doi.org/10.1126/science.1241224

3. Zielinski, M. R., \& Krueger, J. M. (2011). Sleep and innate immunity. Frontiers in Bioscience (Scholar edition), 3, 632-642. https://doi.org/10.2741/s176

4. Matricciani, L., Olds, T., \& Petkov, J. (2012). In search of lost sleep: Secular trends in the sleep time of school-aged children and adolescents. Sleep Medicine Reviews, 16(3), 203-211. https://doi.org/10.1016/j.smrv.2011.03.005

5. Chaput, J.-P., Gray, C. E., Poitras, V. J., Carson, V., Gruber, R., Olds, T., Weiss, S. K., Gorber, S. C., Kho, M. E., Sampson, M., Belanger, K., Eryuzlu, S., Callender, L., \& Tremblay, M. S. (2016). Systematic review of the relationships between sleep duration and health indicators in school-aged children and youth. Applied Physiology, Nutrition \& Metabolism, 41, S266-S282. https://doi.org/10.1139/apnm-20150627

6. Geiger, A., Achermann, P., \& Jenni, O. G. (2010). Association between sleep duration and intelligence scores in healthy children. Developmental Psychology, 46(4), 949-954.

https://doi.org/10.1037/a0019679

7. Buckhalt J. A. (2011). Children's sleep, sleepiness, and performance on cognitive tasks. WMF press bulletins, 2011(2), 1-12.

8. Beebe, D. W. (2011). Cognitive, behavioral, and functional consequences of inadequate sleep in children and adolescents. The Pediatric Clinics of North America, 58(3), 649-665. https://doi.org/10.1016/j.pcl.2011.03.002

9. Bernier, A., Beauchamp, M. H., Bouvette, T. A., Carlson, S. M., \& Carrier, J. (2013). Sleep and cognition in preschool years: Specific links to executive functioning. Child Development, 84(5), 1542-1553. https://doi.org/10.1111/cdev.12063 
10. Walker, M. P. (2008). Cognitive consequences of sleep and sleep loss. Sleep Medicine, 9(Suppl1), S29-S34. https://doi.org/10.1016/S1389-9457(08)70014-5

11. Palmer, C. A., \& Alfano, C. A. (2017). Sleep and emotion regulation: An organizing, integrative review. Sleep Medicine Reviews, 31, 6-16. https://doi.org/10.1016/j.smrv.2015.12.006

12. Reigstad, B., Jørgensen, K., Sund, A. M., \& Wichstrøm, L. (2010). Prevalences and correlates of sleep problems among adolescents in specialty mental health services and in the community: What differs? Nordic Journal of Psychiatry, 64(3), 172-180. https://doi.org/10.3109/08039480903282392

13. Kahn, M., Sheppes, G., \& Sadeh, A. (2013). Sleep and emotions: Bidirectional links and underlying mechanisms. International Journal of Psychophysiology, 89(2), 218-228.

https://doi.org/10.1016/j.ijpsycho.2013.05.010

14. Mindell, J. A., Sadeh, A., Kwon, R., \& Goh, D. Y. T. (2015). Relationship between child and maternal sleep: A developmental and cross-cultural comparison. Journal of Pediatric Psychology, 40(7), 689696. https://doi.org/10.1093/jpepsy/jsv008

15. Gruber, R., Somerville, G., Wells, S., Keskinel, D., \& Santisteban, J. A. (2018). An actigraphic study of the sleep patterns of younger and older school-age children. Sleep Medicine, 47, 117-125. https://doi.org/10.1016/j.sleep.2018.03.023

16. Guglielmo, D., Gazmararian, J. A., Chung, J., Rogers, A. E., \& Hale, L. (2018). Racial/ethnic sleep disparities in US school-aged children and adolescents: A review of the literature. Sleep health, 4(1), 68-80. https://doi.org/10.1016/j.sleh.2017.09.005

17. Staton, S., Rankin, P. S., Harding, M., Smith, S. S., Westwood, E., LeBourgeois, M. K., \& Thorpe, K. J. (2020). Many naps, one nap, none: A systematic review and meta-analysis of napping patterns in children 0-12 years. Sleep Medicine Reviews, 50, https://doi.org/10.1016/j.smrv.2019.101247

18. Weaver, M. D., Sletten, T. L., Foster, R. G., Gozal, D., Klerman, E. B., Rajaratnam, S. M., ... Roenneberg, T. (2021). Adverse impact of polyphasic sleep patterns in humans: Report of the National Sleep Foundation sleep timing and variability consensus panel. Sleep Health, 7(3), 293-302. https://doi.org/10.1016/j.sleh.2021.02.009

19. Jenni, O. G. \& Carskadon, M. A. (2007). Sleep behavior and sleep regulation from infancy through adolescence: Normative aspects. Sleep Medicine Clinics, 2(3), 321-329. https://doi.org/10.1016/j.jsmc.2007.05.001

20. Boergers, J., \& Koinis-Mitchell, D. (2010). Sleep and culture in children with medical conditions. Journal of Pediatric Psychology, 35(9), 915-926. https://doi.org/10.1093/jpepsy/jsq016

21. Nolan, V. G., Gapstur, R., Gross, C. R., Desain, L. A., Neglia, J. P., Gajjar, A., Klosky, J. L., Merchant, T. E., Stovall, M., \& Ness, K. K. (2013). Sleep disturbances in adult survivors of childhood brain tumors. Quality of life research: An international journal of quality of life aspects of treatment, care and rehabilitation, 22(4), 781-789. https://doi.org/10.1007/s11136-012-0208-5

22. Brimeyer, C., Adams, L., Zhu, L., Srivastava, D., Wise, M., Hudson, M., Crabtree, V., Srivastava, D. K., Hudson, M. M., \& Crabtree, V. M. (2016). Sleep complaints in survivors of pediatric brain tumors. Supportive Care in Cancer, 24(1), 23-31. https://doi.org/10.1007/s00520-015-2713-x 
23. Rosen G., Brand S. R., Rosen, G., \& Brand, S. R. (2011). Sleep in children with cancer: case review of 70 children evaluated in a comprehensive pediatric sleep center. Supportive Care in Cancer, 19(7), 985-994. https://doi.org/10.1007/s00520-010-0921-y

24. Lee, S., Narendran, G., Tomfohr-Madsen, L., \& Schulte, F. (2017). A systematic review of sleep in hospitalized pediatric cancer patients. Psycho-Oncology, 26(8), 1059-1069. https://doi.org/10.1002/pon.4149

25. Daniel, L., Kazak, A. E., Li, Y., Hobbie, W., Ginsberg, J., Butler, E., \& Schwartz, L. (2016). Relationship between sleep problems and psychological outcomes in adolescent and young adult cancer survivors and controls. Supportive Care in Cancer, 2, 539. https://doi.org/10.1007/s00520-015-27982

26. Verberne, L. M., Maurice-Stam, H., Grootenhuis, M. A., van Santen, H. M., \& Schouten-van Meeteren, A. Y. N. (2012). Sleep disorders in children after treatment for a CNS tumour. Journal of Sleep Research, 21(4), 461-469. https://doi.org/10.1111/j.1365-2869.2011.00971.x

27. Manley, P. E., McKendrick, K., McGillicudy, M., Chi, S. N., Kieran, M. W., Cohen, L. E., Kothare, S., Scott, R. M., Goumnerova, L. C., Sun, P., London, W., Marcus, K. J., Pomeroy, S. L., \& Ullrich, N. J. (2012). Sleep dysfunction in long term survivors of craniopharyngioma. Journal of Neuro-Oncology, 108(3), 543-549. https://doi.org/10.1007/s11060-012-0859-7

28. Kaleyias, J., Manley, P., \& Kothare, S. V. (2012). Sleep disorders in children with cancer. Seminars in Pediatric Neurology, 19(1), 25-34. https://doi.org/10.1016/j.spen.2012.02.013

29. Steur, L. M. H., Kolk, R. H. E., Mooij, F., de Vries, R., Grootenhuis, M. A., Kaspers, G. J. L., \& Van Litsenburg, R. R. L. (2016). The prevalence and risk factors of sleep problems in pediatric oncology: its effect on quality of life during and after cancer treatment. Expert Review of Quality of Life in Cancer Care, 1(2), 153. https://doi.org/10.1080/23809000.2016.1168265

30. Cheung, Y. T., Brinkman, T. M., Mulrooney, D. A., Mzayek, Y., Liu, W., Banerjee, P., Panoskaltsis, M. A., Srivastava, D., Pui, C., Robison, L. L., Hudson, M. M., Krull, K. R., Panoskaltsis-Mortari, A., \& Pui, C.-H. (2017). Impact of sleep, fatigue, and systemic inflammation on neurocognitive and behavioral outcomes in long-term survivors of childhood acute lymphoblastic leukemia. Cancer, 123(17), 34103419. https://doi.org/10.1002/cncr.30742

31. Van Kooten, J., Maurice-Stam, H., Schouten, A., van Vuurden, D. G., Granzen, B., Gidding, C., ... Grootenhuis, M. A. (2019). High occurrence of sleep problems in survivors of a childhood brain tumor with neurocognitive complaints: The association with psychosocial and behavioral executive functioning. Pediatric Blood \& Cancer 66(11), e27947. https://doi.org/10.1002/pbc.27947

32. Hoskinson, K. R., Wolfe, K. R., Yeates, K. O., Mahone, M., Cecil, K. M., \& Ris, D. (2018). Predicting changes in adaptive functioning and behavioral adjustment following treatment for a pediatric brain tumor: A report form the Brain Radiation Investigative Study Consortium. Psycho-Oncology, 27(1), 178-186. https://doi.org/10.1002/pon.4394

33. Horowitz, T. S., Suls, J., \& Treviño, M. (2018). A call for a neuroscience approach to cancer-related cognitive impairment. Trends in Neurosciences, 41(8), 493-496. 
https://doi.org/10.1016/j.tins.2018.05.001

34. Hirshkowitz, M., Whiton, K., Albert, S. M., Alessi, C., Bruni, O., DonCarlos, L., ... Ware, J. C. (2015). National Sleep Foundation's updated sleep duration recommendations: Final report. Sleep Health, 1(4), 233-243. https://doi.org/10.1016/j.sleh.2015.10.004

35. Smaldone, A., Honig, J. C., \& Byrne, M. W. (2007). Sleepless in America: Inadequate sleep and relationships to health and well-being of our nation's children. Pediatrics-Springfield-, Supp/1, S29. https://doi.org/10.1542/peds.2006-2089F

\section{Figures}

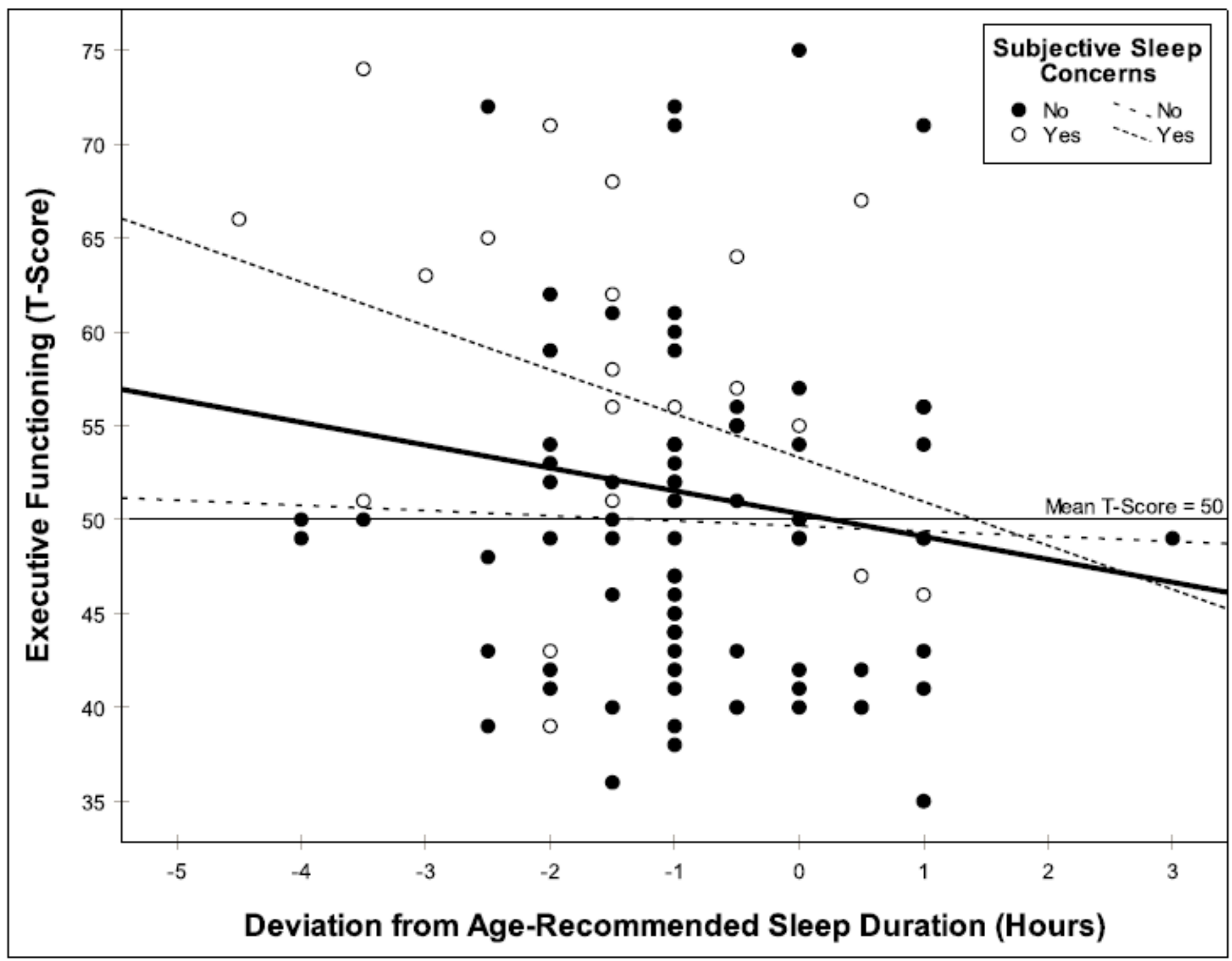

Figure 1

Deviation from age-recommended sleep duration as a predictor of executive functioning among patients with and without subjective sleep concerns 


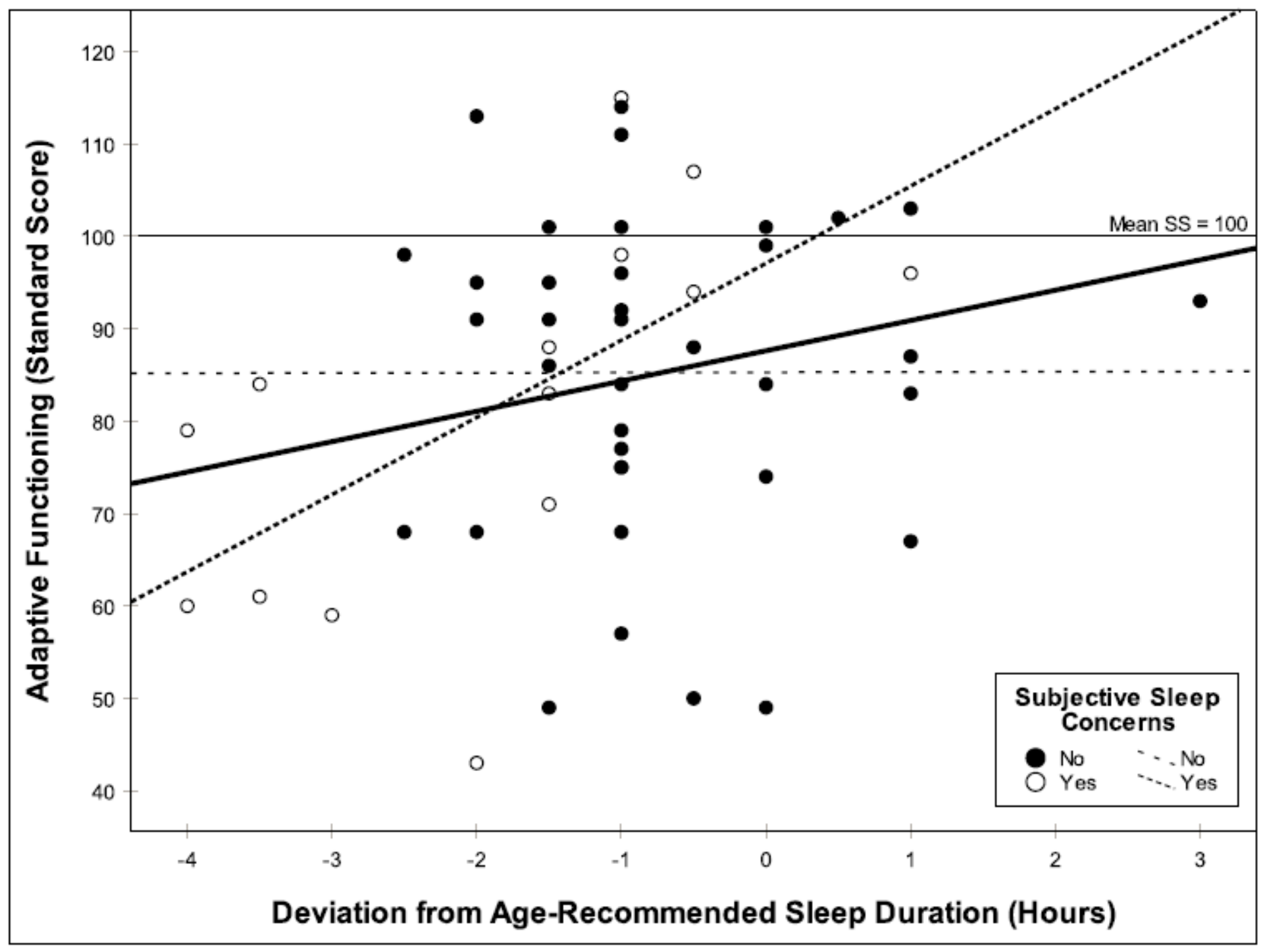

Figure 2

Deviation from age-recommended sleep duration as a predictor of adaptive functioning among patients with and without subjective sleep concerns 